\title{
THE TOPOLOGY AND GEOMETRY OF EMBEDDED SURFACES OF CONSTANT MEAN CURVATURE
}

\author{
WILLIAM H. MEEKS III
}

The mean curvature function $H$ on an oriented surface $M$ in $\mathbf{R}^{3}$ is defined at a point $p$ in $M$ to be

$$
H(p)=\frac{1}{2}\left(\lambda_{1}(p)+\lambda_{2}(p)\right)
$$

where $\lambda_{1}(p)$ and $\lambda_{2}(p)$ are the principal curvatures of $M$ at $p$. When $H$ is constant, $M$ is called a surface of constant mean curvature. A surface is said to have finite type if it is homeomorphic to a closed surface with a finite number of points removed. An important problem in classical differential geometry is the classification of properly embedded finite type surfaces $M$ of constant mean curvature in $\mathbf{R}^{3}$.

If $M$ is a closed embedded surface of constant mean curvature, then it follows from Alexandrov [1] that $M$ must be a round sphere. The classical examples of properly embedded surfaces with zero mean curvature are the plane, the helicoid and the catenoid. Surfaces of zero mean curvature are usually called minimal surfaces. The remaining classical examples of properly embedded surfaces of constant mean curvature were found by Delaunay [4]. The Delaunay surfaces are surfaces of revolution.

Recently Hoffman and Meeks [6, 7] have found examples of properly embedded minimal surfaces which are homeomorphic to closed surfaces of positive genus with 3 points removed. Callahan, Hoffman and Meeks [3] have found other examples with more ends. An annular end $E$ of a properly embedded surface in $\mathbf{R}^{3}$ is a properly embedded annulus $E$ in $M$ where $E$ is homeomorphic to $S^{1} \times[0,1)$. When $M$ has finite type, then every end of $M$ is annular. Hoffman and Meeks have developed a theory to deal with global problems concerning the geometry of properly embedded minimal surfaces $M$ and, in particular, they show that most annular ends of $M$ converge at infinity in $\mathbf{R}^{3}$ to a flat plane or to the end of a catenoid.

Recently N. Kapouleas [8] in his thesis has shown that for every positive integer $k \geq 2$, there exists a properly embedded surface $M_{k}$ of finite type with nonzero mean curvature and with $k$ ends. He also has constructed highergenus examples.

As in the case of minimal surfaces, the annular ends of a properly embedded surface of nonzero constant mean curvature have a special geometry and play an important role in global theorems.

Received by the editors November 14, 1986.

1980 Mathematics Subject Classification (1985 Revision). Primary 53A10.

The research in this paper was partially supported by the following NSF and Department of Energy grants: NSF-DMS-8611574, DE-FG02-86ER25015. 
THEOREM 1. If $M$ is a properly embedded surface of nonzero constant mean curvature, then each annular end of $M$ stays a bounded distance from some ray. ( $A$ ray $r$ is a point set of the form $\left\{t \cdot v \mid t \in \mathbf{R}^{+}\right\}$for some vector $v \in \mathbf{R}^{3}$.)

THEOREM 2. If $M$ is a properly embedded surface of nonzero constant mean curvature, then $M$ is not homeomorphic to a closed surface with exactly one point removed. In particular, $M$ is not a plane.

THEOREM 3. If $M$ is a properly embedded surface of nonzero constant mean curvature and $M$ is homeomorphic to a closed surface punctured in 2 points, then $M$ stays a bounded distance from a straight line.

THEOREM 4. If $M$ is a properly embedded surface of nonzero constant mean curvature and $M$ is homeomorphic to a closed surface punctured in 3 points, then $M$ stays a bounded distance from a plane.

THEOREM 5. Suppose $F$ is a $C^{2}$-foliation of $\mathbf{R}^{3}$ where each leaf of $F$ is a surface of constant mean curvature. Then $F$ consists entirely of parallel planes.

The above theorems are proved by studying stable minimal disks in one of the complements of the surface in $\mathbf{R}^{3}$. The curvature estimates of $R$. Schoen [12] play an essential role in our analysis. The proof of Theorem 5 also depends on results by B. Palmer [10], A. Silveira [11] and Barbosa, Gomes, and Silveira [2].

REMARK. We conjecture that if a properly embedded surface $M$ of constant mean curvature is contained in a half-space of $\mathbf{R}^{3}$, then $M$ has a reflective plane of symmetry which is parallel to the boundary plane of the half-space. The validity of this conjecture has profound consequences when combined with Theorems 2 and 3 given above. The validity of the conjecture and Theorem 2 imply that a properly embedded surface $M$ of constant mean curvature which is homeomorphic to a closed surface punctured in 2 points must be a Delaunay surface, and hence, $M$ is homeomorphic to an annulus.

\section{REFERENCES}

1. A. D. Alexandrov, Uniqueness theorems for surfaces in the large. I, Vestnik Leningrad Univ. Math. 11 (1956), 5-17. (Russian)

2. L. Barbosa, J. Gomes, and A. Silveira, personal communication.

3. M. Callahan, D. Hoffman and W. H. Meeks III, Embedded minimal surfaces with 4 ends, preprint.

4. G. Darboux, Leçons sur la theorie générale des surfaces, Première partie, GauthierVillars, Paris (nouveau tirage), 1941.

5. D. Hoffman and W. H. Meeks III, Complete embedded minimal surfaces of finite total curvature, Bull. Amer. Math. Soc. (N.S.) 12 (1985), 134-135.

6. _ A complete embedded minimal surface in $\mathbf{R}^{3}$ of genus one and three ends, J. Differential Geom. 21 (1985), 109-127.

7.

8. N. Kapouleas, personal communication of thesis results.

9. W. H. Meeks III, The topology and geometry of embedded surfaces of constant mean curvature, preprint. 
10. B. Palmer, Ph.D. Thesis, Stanford University, 1986.

11. A. Silveira, Stable surfaces of constant mean curvature, Ph.D. thesis, IMPA, Rio de Janeiro, Brazil, 1986.

12. R. Schoen, Estimates for stable minimal surfaces in three dimensional manifolds, Seminar on Minimal Submanifolds, Ann. of Math. Studies 103, Princeton Univ. Press, Princeton, N.J., 1983.

Department of MATHEMATICS AND Statistics, University OF MASSAChUSETTS, AMHERST, MASSACHUSETTS 01003 